\title{
Investigating the compliance of COVID-19 protocols in the workplaces of Ardabil, Iran
}

\author{
Asghar Lotfollahzadeh ${ }^{\mathrm{a}}$, Leila Rastgoo ${ }^{\mathrm{a}}$, Islam Shirinzadeh ${ }^{\mathrm{a}}$, \\ Seyedeh Melika Kharghani Moghadam ${ }^{\mathrm{b}}$ and Hossein Ebrahimi ${ }^{\mathrm{c}, *}$ \\ ${ }^{a}$ Ardabil University of Medical Sciences, Ardabil, Iran \\ ${ }^{\mathrm{b}}$ Department of Health Education and Health Promotion, School of Public Health, Iran University of Medical \\ Sciences, Tehran, Iran \\ ${ }^{\mathrm{c}}$ Air Pollution Research Center, Department of Occupational Health, School of Public Health, Iran University \\ of Medical Sciences, Tehran, Iran
}

Received 13 April 2021

Accepted 8 September 2021

\begin{abstract}
.
BACKGROUND: Workplaces are prone to the current outbreak- of COVID-19. Despite the production of the COVID-19 vaccine, due to some challenges in vaccinating all people worldwide, adherence to health protocols is still one of the ways to prevent infection.

OBJECTIVE: This study was conducted with the purpose of investigating the compliance of COVID-19 protocols in the workplaces of Ardabil, Iran.

METHODS: This was a cross-sectional research conducted on the workplaces affiliated to Ardabil University of Medical Sciences between August and September, 2020. A researcher-designed checklist was completed once in early August and once at the end of September for the workplaces. Accordingly, these workplaces were inspected for any compliance with the COVID-19 prevention protocols by health centers during August and September. In addition, the number of patients with COVID-19 was determined for each one of the workplaces.

RESULTS: The results showed that the highest increase was related to screening $(57.8 \%)$ and the lowest change was related to personal hygiene (1.3\%). The rates of increase in performance for small workshops, offices and industries were $35.4 \%$, $33.1 \%$ and $12.4 \%$, respectively. Moreover, a linear and inverse relationship was found between the incidence of COVID-19 and the level of observance of the OVID-19 prevention protocols.

CONCLUSIONS: Based on the results of this study, the inspection made by legal authorities led to the increased commitment of workplace managers to implement prevention programs, thereby increasing the observation level of these protocols in the workplace and reducing the incidence of COVID-19.
\end{abstract}

Keywords: Office, industry, coronavirus, COVID-19, health protocol, inspection

\section{Introduction}

The current outbreak of COVID-19 began in December 2019 in Wuhan, China. People with

\footnotetext{
*Address for correspondence: Hossein Ebrahimi, Air Pollution Research Center, Department of Occupational Health, School of Health, Iran University of Medical Sciences, Tehran, 1449614535, Iran. Tel.: +9886704749; E-mail: hossein.ebrahimi@yahoo.com.
}

COVID-19 mostly present a wide variety of symptoms reported to range from mild symptoms to severe illness. Of note, the common symptoms of COVID19 are fever, dry cough and shortness of breath [1]. People infected with COVID-19 can transmit the virus to others through respiratory droplets, especially during coughing and sneezing. Also, it has been shown that the virus could be transmitted over a distance of 2 meters from an infected person. Moreover, 
the surfaces contaminated with mucus or respiratory droplets of the patient with COVID-19 play an important role in virus transmission [2-4]. Therefore, workplaces are known as a gathering place for people who can expose many employees to COVID-19 $[5,6]$.

In some workplaces, depending on the nature of the work performed in them, workers are forced to work in a close contact with each other or to use common tools and equipment which increase the risk of COVID-19 transmission [7, 8]. Based on each occupational exposure to COVID-19, the Occupational Safety and Health Administration (OSHA) has divided the risk of exposure during performing job tasks into the following four levels: very high, high, moderate, and low. This risk is determined according to the type of work as well as the likelihood of having contact with infected or suspected cases of COVID19 at a distance of 2 meters [9]. Based on this division, the employees of industries and offices are classified in the moderate group.

The end of the current COVID-19 pandemic is uncertain yet [10]. Despite the production of the COVID-19 vaccine, there are still many challenges for an effective and safe program for global vaccination such as initial scarcity of doses and lack of access of all countries and people to the produced vaccine [10]. Therefore, prevention and control measures are still among the most important solutions for the control of COVID-19 [11]. Various organizations such as the OSHA and World Health Organization (WHO) have proposed some recommendations to prevent COVID-19 infection for employees in their workplaces and have recommended that companies should develop a plan to manage coronavirus prevention. The principles of prevention of COVID-19 in workplaces include observing personal hygiene, using personal protective equipment (PPE), doing social distancing, teleworking and employee health/ body temperature screening $[12,13]$. Furthermore, the Ministry of Health, Treatment and Medical Education of Iran has developed COVID-19 prevention protocols in workplace and then made them available to organizations and industries [14]. Among the measures taken in the workplace of Iran to control the release of COVID-19 can be mentioned to telework, employee health/ body temperature screening, using of masks, strengthening ventilation systems, personal hygiene, etc. Given that the priorities in industries and organizations are work and production, despite the development and presentation of COVID19 prevention protocols, the implementation of these
Table 1

Workplace division and sample size of each group

\begin{tabular}{lccc}
\hline & $\begin{array}{c}\text { Banks and } \\
\text { offices }\end{array}$ & Industries & $\begin{array}{c}\text { Small } \\
\text { workshops* }\end{array}$ \\
\hline Number & 831 & 387 & 20888 \\
Sample size & 263 & 187 & 377 \\
\hline
\end{tabular}

*Workplaces with less than 10 employees.

protocols in some cases becomes a victim of production and work. Therefore, in such cases, pressure levers are required to implement these protocols. Furthermore, monitoring the observance of the protocols in industries and organizations by relevant and legal organizations can ensure the proper implementation of them. Hence, this study was aimed to investigate the compliance of COVID-19 prevention protocols in the workplaces affiliated to Ardabil University of Medical Sciences, Ardabil, Iran.

\section{Material and methods}

\subsection{Study population}

This was a cross-sectional research conducted on the workplaces affiliated (under supervision) to Ardabil University of Medical Sciences between August and September, 2020. To determine the sample size, the number of the workplaces in nine cities of Ardabil Province, which are under supervision to Ardabil University of Medical Sciences, was extracted. Thereafter, these workplaces were classified according to the diversity in the nature of work in different workplaces, as shown in Table 1, and the samples were selected from each group based on Krejcie and Morgan's table [15]. Next, the workplaces under each category were randomly selected.

\subsection{Data collection tools}

A researcher-designed checklist was used for data collection. This checklist was designed based on the COVID-19 prevention protocols provided by the Ministry of Health and Medical Education of Iran for workplaces. Correspondingly, this checklist consisted of 14 questions in the following four fields: personal hygiene, PPE, social spacing, and screening (Table 2).

Two qualitative and quantitative research approaches based on the Lawshe model were used to determine the content validity of the checklist [16]. In the qualitative review of the content validity, 15 
Table 2

The items of the designed checklist

\begin{tabular}{ll}
\hline Field & Items \\
\hline Personal hygiene & 1- Procurement of soap and detergents \\
& 2- Provide hand and surface disinfectants at various workplace locations \\
& 3- Personalize phone, desk, chair and more \\
& 4- Existence of face recognition system instead of fingerprint for record the presence of employees \\
& 1- Provide breathing masks for workers (2 masks per shift) \\
Personal protective equipment & 1- Teleworking \\
Social spacing & 2- Proper physical distance (at least 2 meters) between workers in workstations \\
& 3- Proper physical distance with customers \\
& 4- Consider a special isolation room to house workers suspected of COVID-19 \\
& 5- Sessions hold as a video conference \\
& 1- Temperature check \\
Screening & 2- Oxygen saturation check \\
& 3- Prevent the entry of clients without masks \\
& 4- Restrictions on the use of canteens and restaurants \\
\end{tabular}

experts in the field of health and safety were invited to participate as a panel group. Afterward, two indicators, including content validity ratio (CVR) and content validity index (CVI) were used for the quantitative review of the content validity. For this purpose, the CVR index was used to ensure that the most important and accurate contents (item necessity) were selected, and the CVI was also utilized to ensure that the checklist items were used to measure exactly the desired content in the best way $[16,17]$. The minimum acceptable values of CVI and CVR were 0.79 and 0.49 , respectively [18].

Reliability means that the measurement tool gives the same results under the same conditions. In this study, the common method, Cronbach's alpha, was used to measure reliability [17]. According to the current research's purpose, to determine the reliability, the designed questionnaire was completed in the workplaces affiliated to Ardabil University of Medical Sciences.

\subsection{Methods}

The designed checklist was completed for the selected workplaces during sudden visits by inspectors of health centers in early August 2020. The checklists were completed by a researcher who was a member of the inspection team. Besides, these workplaces were inspected for any compliance with the protocols provided by health centers between August and September. The designed checklist was then completed for the selected workplaces again at the end of September. In addition, the number of patients with COVID-19 was determined once at the beginning of August and once at the end of September for the selected workplaces. Finally, the completed
Table 3

The performance of the workplaces in implementing the COVID-19 prevention protocols

\begin{tabular}{lcc}
\hline $\begin{array}{l}\text { Dimensions of COVID-19 } \\
\text { prevention protocol }\end{array}$ & $\begin{array}{c}\text { August } \\
\text { (Mean } \pm \text { S.D) }\end{array}$ & $\begin{array}{c}\text { September } \\
(\text { Mean } \pm \text { S.D) }\end{array}$ \\
\hline Personal hygiene & $68.1 \% \pm 0.7 \%$ & $69.4 \% \pm 0.5 \%$ \\
Personal protective & $28.0 \% \pm 1.8 \%$ & $48.6 \% \pm 1.7 \%$ \\
$\quad$ equipment & & \\
Screening & $20.0 \% \pm 1.5 \%$ & $77.8 \% \pm 2.8 \%$ \\
Social spacing & $26.6 \% \pm 2.2 \%$ & $78.8 \% \pm 3.4 \%$ \\
\hline
\end{tabular}

checklists were analyzed for two time periods and the results were compared with each other. Additionally, the results obtained from the checklists were compared with the incidence of COVID-19.

\section{Results}

According to the validation results of the checklist, the lowest and highest values of the CVI were equal to 0.82 and 0.98 , respectively. Moreover, the lowest and highest CVR values were 0.87 and 1, respectively. Notably, the total values of CVI and CVR were achieved as 0.88 and 0.98 , respectively. Also, the value of Cronbach's alpha coefficient of the questionnaire was calculated as 0.782 .

Table 3 shows the performance of the studied workplaces in the implementation of the COVID19 prevention protocols. The highest increase was found to be attributable to screening and the lowest change was related to personal hygiene. The extent of change in the dimensions of the COVID-19 prevention protocols by the workplaces is shown in Fig. 1. The highest and lowest levels of increases in performance belonged to small workshops and industries, respectively. According to the results obtained, the 


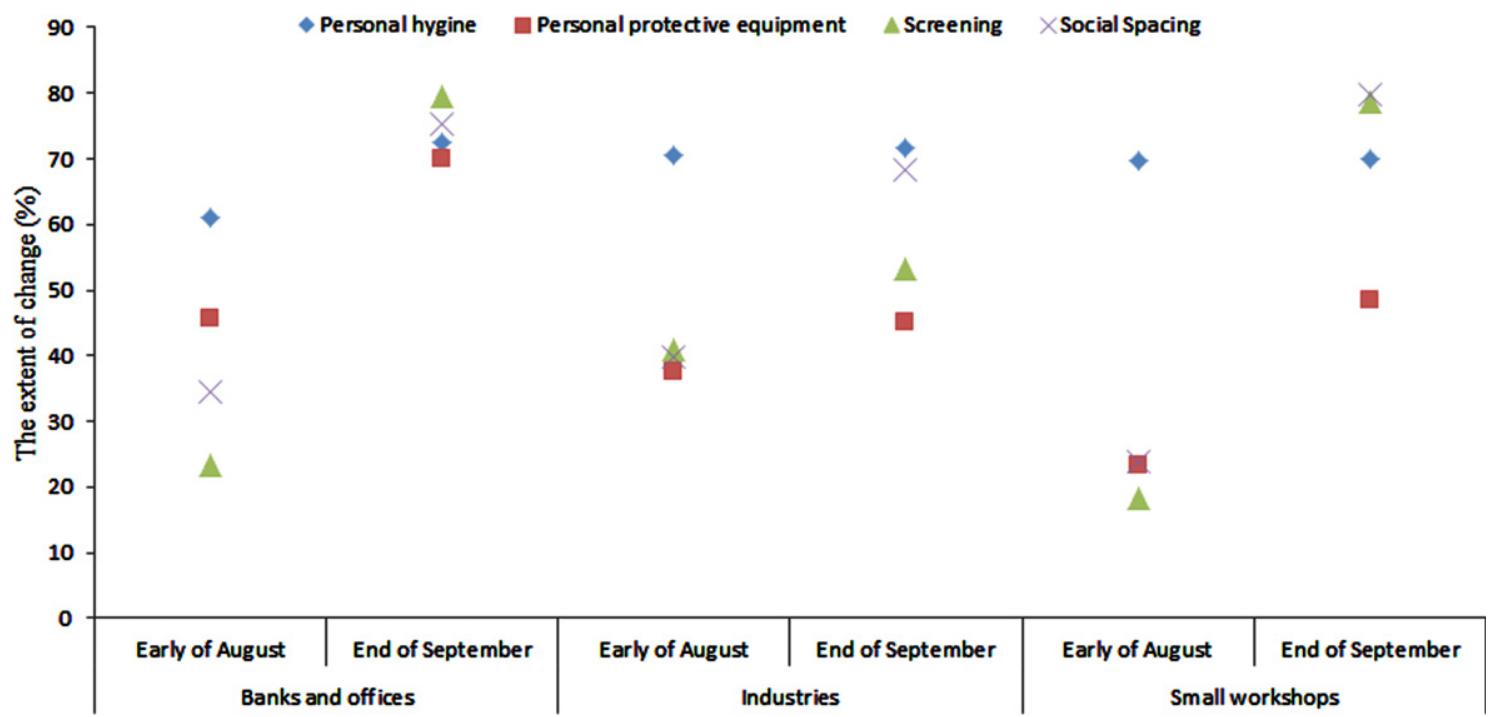

Fig. 1. The extent of change in the dimensions of the COVID-19 prevention protocols by the workplaces.

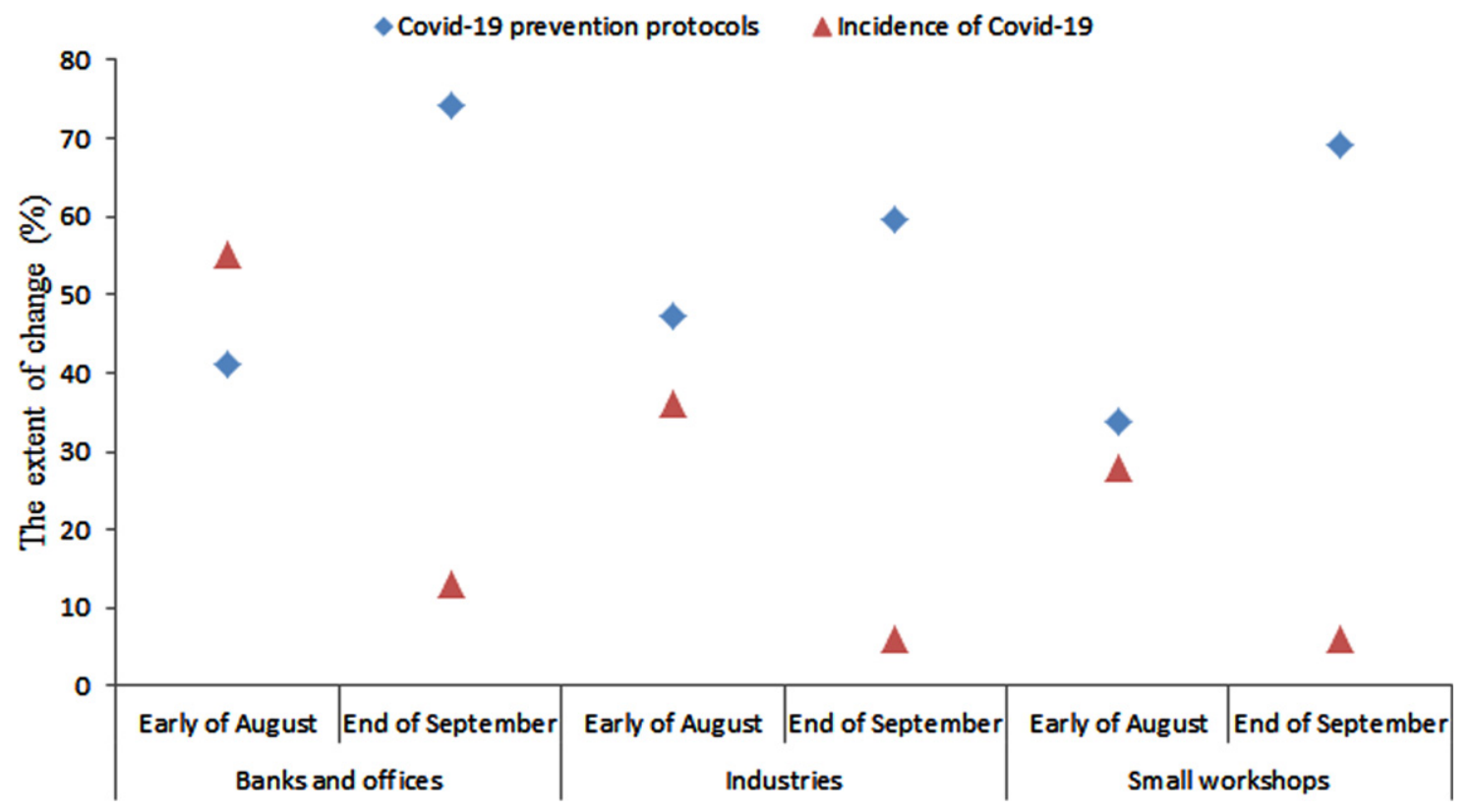

Fig. 2. The relationship between performance of workplaces in the field of COVID-19 prevention protocols and the incidence of COVID-19.

rates of increase in performance for small workshops, offices, and industries were $35.4 \%, 33.1 \%$ and $12.4 \%$, respectively.

Figure 2 shows the relationship between the performance of workplaces in terms of the COVID-19 prevention protocols and the incidence of COVID-19, a linear and inverse relationship was found between the incidence of COVID-19 and the level of observance of the COVID-19 prevention protocols.

\section{Discussion}

The objective of this study was to investigate the compliance of COVID-19 prevention protocols in the workplaces affiliated to Ardabil University of Medical Sciences, Ardabil, Iran. According to a study conducted by the American Association of Tactical Operations Committee, the data collection and assessment processes on the respondents' 
preparedness levels using a standardized questionnaire are needed for organizations to control a catastrophic event such as a terrorist attack or a natural disaster [19, 20]. Furthermore, it is essential to design and develop a checklist for evaluating the performance of COVID-19 prevention protocols in workplaces. Based on the results of the design and validation phases of the checklist, it was found that the designed checklist had good validity and reliability. Moreover, the design checklist benefited from a good efficiency for data collection regarding the management of COVID-19 prevention protocols.

The findings of the study showed that the investigated workplaces had poor performance at the beginning of August, but their performance increased up to the end of September due to the inspection made by the health centers. Planning and preparedness to deal with the COVID-19 crisis are currently considered as the national and international necessities. Thus, these should be paid special attention by policy makers and health officials in adopting preventive behaviors at the community level in order to control the COVID-19 pandemic [21].

The Ministry of Health and Medical Education of Iran had developed health protocols to prevent COVID-19 in workplaces [22]. However, this required management commitment to implement the developed programs. In workplaces, the priority is to manage both production and productivity, and there is not much inclination to be invested on other aspects. Commitment to implement the developed protocols can be built in managers through the legislation and supervision of legal authorities.

The findings of the current study indicated that the highest rates of increase in performance belonged to screening, social spacing, PPE and personal hygiene. According to the WHO, some of the most important behaviors to prevent COVID-19 infection are as follows: regular hand washing, personal hygiene, and avoiding from shaking hands and hugging [23].

The level of personal hygiene, including frequent hand washing and the use of disinfectants, was better than the other dimensions at the beginning of the study, and this factor had the least increase throughout performing the study. In interpreting this result, much attention should be paid to the fact that the culture of observing personal hygiene in the field of COVID-19 prevention has been previously built in the community and family through national education; therefore, it had a favorable situation at the beginning of this study.
It was reported that COVID-19 is transmitted through airborne particles, particularly indoors with insufficient ventilation [24]. Therefore, there is a possibility of getting coronavirus through touching an infected object or surface [25]. Hence, the application of PPE such as respirator masks and gloves can be effective on preventing this infection. Moreover, two aspects of providing PPE and creating a culture for using these devices should be considered in the field of using PPE in the workplace. Accordingly, providing suitable PPE is affected by management commitment and the increased management commitment consequently leads to the increased provision of PPE.

When looking at the statistics, although the majority of deaths and infections caused by COVID-19 are related to people aged over 50 years old and those with underlying diseases, there is still a risk of infection in the whole population and the importance of prevention is increasing due to asymptomatic carriers, because these people can transmit the disease to other population groups [26]. Therefore, screening both employees and clients can be effective on the prevention of the disease, as asymptomatic carriers can be identified and then their entrance to the workplace can be forbidden.

The reproduction rate number is a very important component in terms of virus transmission. Accordingly, reproductive rate refers to the average number of people who are at risk of contracting the disease by an infected person [27]. Thus, reproduction rate is high in these places due to the population density in the workplace. In this regard, teleworking and distancing are considered as the best ways to reduce the reproduction rate in the workplace. According to the results of this study, the largest increase was related to social spacing.

Management commitment is very important in implementing this dimension, given that social spacing may result in the reduced production or high costs required to implement social spacing. It was found that monitoring performed by legal authorities by increasing the commitment of management can increase compliance with COVID-19 protocols in the workplace.

It should be pointed out that, at the beginning of the study, industries were under the best condition and small workshops were under the worst one. It is worth mentioning that all industries covered by Ardabil University of Medical Sciences had an occupational health expert. Notably, one of the tasks of these people is to implement the COVID-19 protocols 
in the workplace. Lack of knowledge in people on the ways of transmission and prevention of this emerging disease is one of the main causes of the spread of coronavirus. As a result, education is a key element in implementing the required prevention programs. It is impossible to achieve program development, preparation, and procurement of high-performance prevention materials and tools without any proper training. Therefore, it is of great importance to hold training courses on ways of transmitting and prevention of coronavirus in workplaces. It should be noted that most of the surveyed industries had held some related training courses. Moreover, the highest and lowest increase rates in performance during the study belonged to small workshops and industries, respectively. The inspection had the least effect on increasing the performance of the industries due to the large number of employees and the more complex work environment in these workplaces, despite the presence of an occupational health expert and the holding some training courses. Also, there was a linear and inverse relationship between the incidence of COVID-19 and the observance of COVID-19 protocols, in such a way, that by increasing the observance level of COVID-19 protocols during the study, the incidence of COVID-19 in the workplace reduced. Observing personal hygiene and using a proper diet are considered as the most important ways to fight COVID-19 [28, 29]. Some of the best strategies to deal with the coronavirus are improving personal hygiene, using medical masks, having adequate rest, and utilizing a proper ventilation system [30]. The results of this study indicated that observing COVID19 protocols can reduce the incidence of this disease in the current situation where there is no definitive treatment as well as no approved vaccine to prevent COVID-19 yet. Comparing the results before August and after September, it can be said that inspections by health centers play a key role in the implementation of COVID-19 prevention protocols in the workplace.

The small study interval and a large number of confounding variables were the major limitations of the present study.

\section{Conclusion}

The results of this study showed that the inspection made by health centers could result in the increased commitment of workplace managers to implement COVID-19 protocols, which, consequently, increased the implementation level of these protocols in the workplace. Furthermore, a linear and inverse relationship was found between the incidence of COVID-19 and the level of observance of the COVID-19 prevention protocols. Therefore, in the current situation that there is no certain, effective medicine or anti-virus vaccine to treat or preventCOVID-19, observing the COVID-19 protocols in the workplace can reduce the incidence of COVID-19.

\section{Acknowledgments}

The authors would like to thank all workplace managers and employees who participated in the study.

\section{Conflict of interest}

The authors declare that there is no conflict of interest regarding the publication of this article.

\section{References}

[1] Sobral J, Teixeira D, Morais H, Neves M. Methodology to assess medical processes based on a Failure Mode and Effects Analysis (FMEA). 2017 IEEE 5th Portuguese Meeting on Bioengineering (ENBENG): IEEE; 2017. p. 1-4.

[2] Dhand R, Li J. Coughs and sneezes: their role in transmission of respiratory viral infections, including SARSCoV-2. American Journal of Respiratory and Critical Care Medicine. 2020;202:651-9.

[3] Arefi MF, Poursadeqiyan M. A review of studies on the epidemic crisis of COVID-19 disease with a preventive approach. Work. 2020(Preprint):1-3.

[4] Soheili S, Shariat A, Anastasio A. Modification of existing occupational therapeutic protocols in response to new normal life after COVID 19: Letter to the Editor. Work. 2020 Jul 9(Preprint):1-2.

[5] de Lucas Ancillo A, del Val Núñez MT, Gavrila SG. Workplace change within the COVID-19 context: a grounded theory approach. Economic Research-Ekonomska Istraživanja. 2020:1-20.

[6] Kim E-A. Social Distancing and Public Health Guidelines at Workplaces in Korea: Responses to Coronavirus Disease19. Safety and Health at Work. 2020;11:275-83.

[7] Bielicki JA, Duval X, Gobat N, Goossens H, Koopmans $\mathrm{M}$, Tacconelli E, et al. Monitoring approaches for healthcare workers during the COVID-19 pandemic. The Lancet Infectious Diseases. 2020.

[8] Ebrahimi H, Yarahmadi R, Kharghani Moghadam SM, Malekpour F, Nasezadeh Z, Taheri E, et al. Investigating the status of prevention of coronavirus in industries. Iran Occupational Health. 2020;17:1-10.

[9] Safety O, Administration H. Guidance on preparing workplaces for COVID-19. US: Department of Labor. 2020. 
[10] Abd El-Aziz TM, Stockand JD. Recent progress and challenges in drug development against COVID-19 coronavirus (SARS-CoV-2)-an update on the status. Infection, Genetics and Evolution. 2020:104327.

[11] De Hert M, Mazereel V, Detraux J, Van Assche K. Prioritizing COVID-19 vaccination for people with severe mental illness. World Psychiatry. 2021 Feb;20(1):54.

[12] Sheppard J, Thomas CB. Community pharmacists and communication in the time of COVID-19: applying the health belief model. Research in Social and Administrative Pharmacy. 2020.

[13] Penteado CSG, de Castro MAS. COVID-19 effects on municipal solid waste management: What can effectively be done in the Brazilian scenario? Resources, Conservation and Recycling. 2020;164:105152.

[14] Organization WH. Getting your workplace ready for COVID-19: How COVID-19 spreads, 19 March 2020. World Health Organization; 2020.

[15] Emadi S-N, Ehsani AH, Balighi K, Nasimi M. Cosmetic surgeries amidst pandemic area of COVID-19: suggested protocol. Journal of Dermatological Treatment. 2020:1-2.

[16] Kotrlik J, Higgins C. Organizational research: Determining appropriate sample size in survey research appropriate sample size in survey research. Information Technology, Learning, and Performance Journal. 2001;19:43.

[17] Newman I, Lim J, Pineda F. Content validity using a mixed methods approach: Its application and development through the use of a table of specifications methodology. Journal of Mixed Methods Research. 2013; 7:243-60.

[18] Polit DF, Beck CT, Owen SV. Is the CVI an acceptable indicator of content validity? Appraisal and recommendations. Research in Nursing \& Health. 2007;30:459-67.

[19] Vakili MM, Jahangiri N. Content validity and reliability of the measurement tools in educational, behavioral, and health sciences research. Journal of Medical Education Development. 2018;10(28):106-18.

[20] Wynd CA, Schmidt B, Schaefer MA. Two quantitative approaches for estimating content validity. Western Journal of Nursing Research. 2003;25:508-18.
[21] Mohammadfam I, Kamalinia M, Momeni M, Golmohammadi R, Hamidi Y, Soltanian A. Evaluation of the quality of occupational health and safety management systems based on key performance indicators in certified organizations. Safety and Health at Work. 2017;8:156-61.

[22] Wu Z, McGoogan JM. Characteristics of and important lessons from the coronavirus disease 2019 (COVID-19) outbreak in China: summary of a report of 72314 cases from the Chinese Center for Disease Control and Prevention. Jama. 2020;323:1239-42.

[23] Raeisi A, Tabrizi JS, Gouya MM. IR of Iran National Mobilization against COVID-19 Epidemic. Archives of Iranian Medicine. 2020;23:216.

[24] Organization WH. Coronavirus disease 2019 (COVID-19): situation report, 82. 2020.

[25] Hwang SE, Chang JH, Oh B, Heo J. Possible aerosol transmission of COVID-19 associated with an outbreak in an apartment in Seoul, South Korea, 2020. International Journal of Infectious Diseases. 2021;104:73-6.

[26] Grover S. Frequently Asked Questions (FAQs) about Coronavirus Disease 2019 (COVID-19). Epidemiology International (E-ISSN: 2455-7048). 2020;5:14-21.

[27] Rothan HA, Byrareddy SN. The epidemiology and pathogenesis of coronavirus disease (COVID-19) outbreak. Journal of Autoimmunity. 2020:102433.

[28] Dietz K. The estimation of the basic reproduction number for infectious diseases. Statistical methods in medical research. 1993;2:23-41.

[29] Klemeš JJ, Van Fan Y, Jiang P. The energy and environmental footprints of COVID-19 fighting measures-PPE, disinfection, supply chains. Energy. 2020;211:118701.

[30] Dave D, Friedson AI, Matsuzawa K, Sabia JJ. When do shelter-in-place orders fight COVID-19 best? Policy heterogeneity across states and adoption time. Economic Inquiry. 2021;59:29-52.

[31] Siddiqui AA, Alshammary F, Amin J, Rathore HA, Hassan I, Ilyas M, Khursheed Alam M. Knowledge and practice regarding prevention of COVID-19 among the Saudi Arabian population. Work. 2020 Jan 1(Preprint):1-9. 